\section{Dados de epidemiologia descritiva de transtornos mentais em grupos populacionais do Brasil}

\author{
Anastácio Ferreira Morgado* \\ Evandro da Silva Freire Coutinho*
}

*Escola Nacional de Saúde Pública - FIOCRUZ·RJ.
Neste trabalho são apresentados três tipos de dados sobre transtornos mentais: taxas de prevalência em amostras representativas, taxas de prevalência em estudos de familias $e o$ indicador de morbidade proporcional, isto é, de proporções por diagnóstico entre pacientes internados em hospitais psiquiátricos do Brasil.

Em relação a um inquérito epidemiológico, realizado em amostra representativa, a taxa de prevalência total foi de $20 \%$, da qual 3,0\%, 14,6\%, 0,7\%,1,1\% e 0,5\% foram de alcoolismo, neuroses, psicoses, oligofrenia e sindrome orgânica do cérebro, respectivamente. A distribuição por sexo mostrou uma clara e esperada predominância de alcoolismo nos homens e de neuroses nas mulheres.

Em um estudo voltado para a familia de origem (pais $e$ irmãos) de 150 grandes consumidores de drogas, a taxa de prevalência total entre 796 familiares foi de 23,2\%. As taxas especificas de problemas associados ao álcool, dependência de drogas, psicoses, neuroses, epilepsia, transtorno anti-social da personalidade e outros diagnósticos foram, respectivamente, de 8,4\%; 5,3\%; 3,1\%; 4,0\%;0,8\%; 0,9\% e $0,8 \%$. Os problemas associados ao álcool predominaram nos pais e neuroses nas mães, enquanto dependência de drogas predominou nos irmãos dos 150 consumidores-indice.

$O$ perfil da assistência psiquiátrica brasileira é parcialmente visto através do indicador de proporções de primeiras internações nos hospitais psiquiátricos nacionais. Esse indicador é distribuido por diagnóstico, em série histórica, desde 1940 até 1977. Tais dados mostram que as primeiras internações têm aumentado nas últimas quatro décadas, com maiores proporções dos diagnósticos de esquizofrenia e alcoolismo e dependência de drogas. Há, porém, diferença nas proporções de diagnóstico entre 0 hospital público e o particular-conveniado; tal diferença e o referido aumento de primeiras internações são de suma importância para os programas da area de Saúde Mental.

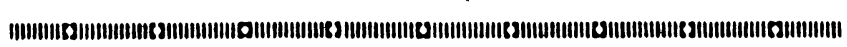

Recebido para publicação em $18 / 03 / 85$. 


\section{INTRODUÇÃO}

No presente trabalho, apresentam-se taxas de prevalência de transtomos mentais referentes a alguns grupos populacionais do Brasil. Não são numerosos os trabalhos que fornecem dados suficientes para o cálculo de taxas, mas um cuidadoso aproveitamento daqueles existentes contribui muito para o dimensionamento dos principais transtomos mentais em nosso meio.

Achamos que uma articulação desses poucos estudos com as taxas de prevalência de amostras seletivas e, num terceiro escalonamento, com a morbidade internada proporcional por diagnóstico, dá até mesmo para o referido dimensionamento alcançar o nível nacional. Com referência a esse último indicador, há importantes dados para o Brasil como um todo. Embora não sejam de prevalência, os números relativos à demanda internada em hospitais psiquiátricos constituem o melhor indicador disponível da epidemiologia psiquiátrica em nosso meio.

De fato, há bons dados de 1940 para cá, o que permite acompanhar as mudanças na morbidade internada em hospitais psiquiátricos por quase quatro décadas. Com pequenas aproximações nas categorias de diagnóstico, vários resultados podem ser comparados.

Uma coisa precisa ficar bem clara: as taxas de transtornos mentais somente têm sentido quando interpretadas à luz dos principais fatores que as condicionam ou thes produzem grandes interferências. Essa interpretação é imprescindivel para explicar tanto as diferenças dos próprios dados de um estudo particular, quanto as diferenças em relação às taxas esperadas, obtidas em estudos internacionais.

Quais são os principais fatores condicionantes das diferenças nos resultados das taxas de prevalência de transtornos mentais? O primeiro e mais importante deles é a própria conceituação de cada um desses transtornos. De fato, as divergências na definição do que seja "caso" desse ou daquele transtomo são tantas e profundas, a ponto de questionar-se muito a validade da comparação dos resultados dos diferentes estudos. Os dois estudos abaixo ilustram isso de uma forma incontestável, uma vez que foram realizados quase na mesma época, em populações semelhantes, com similar período de coleta e, em ambos, empregou-se exatamente o mesmo questionário; porém, os resultados de um foram completamente diferentes daqueles do outro.

Um dos estudos foi realizado por Srole e col. ${ }^{23}$, no qual entrevistaram diretamente uma amostra de população urbana dos EUA, de 20 a 59 anos de idade. A taxa de prevalência total por eles encontrada foi de $23,4 \%$. O segundo dos dois estudos foi realizado por Manis e col. ${ }^{15}$, no qual 
também entrevistaram diretamente uma amostra de população urbana dos EUA, igualmente de 20 a 59 anos de idade. Porém, nesse estudo, os autores encontraram uma taxa de prevalência total de apenas $3,4 \%$ ! Isto é, nesse estudo, diagnosticaram-se transtomos mentais quase sete vezes menos que naquele de Srole e col. ${ }^{23}$.

Tendo em conta que as variáveis reais, responsáveis por diferenças verdadeiras, foram comuns a esses dois estudos, a enorme diferença em seus resultados necessariamente decorreu de algum artefato de pesquisa - num ou noutro estudo, dependendo da escola doutrinária que se abrace. Srole e col. ${ }^{23}$ adotaram critério amplo de inclusão de casos, enquanto Manis e col. ${ }^{15}$ empregaram definição bem restrita de cada transtorno, de sorte que incluíram somente os casos graves. A grande diferença das taxas totais desses estudos não é real, e sim artificial - meramente um efeito da divergência conceitual do que seja "caso" de transtorno mental. Em qualquer estudo de epidemiologia psiquiátrica, é suprema a importância da conceituação de caso. Os problemas em torno dessa conceituação e as conseqüências da adoção de diferentes critérios de inclusão como sendo caso estão bem descritos no trabalho de Blum ${ }^{4}$ e no de Williams e col. ${ }^{26}$, ambos orientados para estudos epidemiológicos em psiquiatria.

Além dos critérios de diagnóstico, ao compararem-se resultados de diferentes estudos, importa muito atentar-se para a influência das seguintes variáveis:

a) Rigor na procura dos casos. A entrevista direta, pessoal, da amostra do estudo, identifica mais casos do que qualquer método indireto - registro de ambulatórios ou hospitais, por exemplo. Isto porque uma fonte de dados, mesmo que registre todos os casos oriundos de uma população definida, será sempre um sub-registro, pois um importante subconjunto de casos por uma ou outra razão não busca o tratamento. Dohrenwend e Dohrenwend 10 acham que essa variável juntamente com aquela do conceito de caso são as principais responsáveis pelas diferenças nos estudos de prevalência de transtornos mentais. No citado trabalho, efetuou-se uma revisão de 35 estudos de prevalência em populações, nos quais a taxa total variou de 0,8 a $64,0 \%$ !

b) Idade da população. Em geral, os transtornos mentais são crônicos e vão se acumulando com o aumento da idade. Se na amostra há elevada proporção de jovens, tende-se a subestimar a prevalência total.

c) Composição da amostra. Se esta não obedecer à estrutura da população quanto a sexo, idade, estrato sócio- 
econômico, situação conjugal e inserção ocupacional, necessariamente fornecerá um resultado questionável. Uma amostra onde predomine o sexo feminino, por exemplo, fornecerá baixa prevalência de problemas associados ao consumo de álcool e outras drogas e, ao contrário, superestimará a taxa de PMD e de uma ou outra neurose. Se na composição da amostra houver uma concentração de idosos, inativos e aposentados, não será surpresa encontrarem-se elevadas taxas de depressão, suicídio e de doenças degenerativas do S.N.C.

d) Outras variáveis. O tipo de questionário empregado, a formação dos entrevistadores, a aceitação do estudo pela população e até a hora em que se realiza a coleta de dados são fatores que também interferem nos resultados.

Naturalmente que as ressalvas acima são fundamentais para se discutirem os resultados que a seguir serão apresentados.

A maior parte dos dados apresentados neste trabalho é voltada para o indicador de morbidade proporcional, referente à primeira internação do paciente em hospital psiquiátrico. Procurou-se aproveitar todos os estudos disponíveis que fornecessem grandes números, a partir de 1940. Mesmo quando um dado estudo fornecia aquele indicador, ele foi recalculado. Para isso, sempre se ateve ao exato valor absoluto expresso nos resultados de cada autor.

É inegável que os dados de diagnóstico foram gerados em hospitais que tinham diferenças nos procedimentos de formulação do diagnóstico psiquiátrico. Contudo, isso não justifica o não-uso de tais dados; em grande número, essas diferenças são atenuadas, e o conjunto dos vários resultados dá para compor a evolução da demanda que pela primeira vez ingressa num hospital psiquiátrico.

\section{ALGUNS ESTUDOS DE PREVALÊNCIA}

Em grupos populacionais do Brasil, como já dissemos, genuínos estudos de prevalência são de fato poucos. Arrolamos os estudos de Coutinho ${ }^{9}$, Moura Fé e col. ${ }^{18}$; Santa$\mathrm{na}^{22}$, Caetano ${ }^{6}$, Morgado $^{17}$, e Almeida Filhor.

O trabalho de Santana 22 é particularmente ilustrativo dessas poucas contribuições nacionais. Em síntese, realizouse um inquérito epidemiológico em amostra representativa de uma população de 26.212 habitantes de um bairro de Salvador, Bahia. A coleta foi direta, com entrevista pessoal das 1.531 pessoas da amostra: na primeira etapa, rastrearamse os suspeitos de transtorno mental e, numa segunda etapa, estes foram examinados por psiquiatras. Entre vários resul- 
tados, apresentamos, na tabela 1 , os referentes ao diagnóstico psiquiátrico.

TABELA 1

Taxas de prevalência (T.P.) por doença mental especifica e segundo sexo. Salvador, BA. 1977

\begin{tabular}{lccccrr}
\hline \multicolumn{1}{c}{ Sexo } & Feminimo & $(\mathrm{N}=837)$ & Masculino & $(\mathrm{N}=694)$ & Total & $(\mathrm{N}=1531)$ \\
& No & TP (\%) & No & TP (\%) & No & TP (\%) \\
Diagnóstico & & & & & & \\
& $639^{*}$ & 76,3 & $586^{*}$ & 84,4 & 1225 & 80,0 \\
Sem anormalidades & $198^{*}$ & 23,7 & $108^{*}$ & 15,6 & 306 & 20,0 \\
Com anormalidades & 6 & 0,7 & 40 & 5,8 & 46 & 3,0 \\
$\quad$ Alcoolismo & 174 & 20,8 & 50 & 7,2 & 224 & 14,6 \\
Neurose & 3 & 0,4 & 8 & 1,2 & 11 & 0,7 \\
Psicose & 11 & 1,3 & 6 & 0,9 & 17 & 1,1 \\
Oligofrenta & 4 & 0,5 & 4 & 0,6 & 8 & 0,5 \\
Sind. Org. Cerebral & 837 & 24,0 & 694 & 16,0 & 1531 & 20,0 \\
\hline Total & & & &
\end{tabular}

Fonte: Adaptação de tabela de Santana 22 (pág. 77)

* $\left(\mathrm{X}_{\mathrm{c}}^{2}=15,04 ; \mathrm{p}<0,001\right)$

Como se observa na tabela 1 , a prevalência total foi de $20 \%$, com o esperado predomínio do diagnóstico de neurose. $\mathrm{Na}$ comparação entre os sexos, detectou-se o predomínio do alcoolismo no sexo masculino e o de neurose no sexo feminino. Von Zerssen e Weyerer ${ }^{25}$ consideram que essa antiga questão é instrutiva, quando se leva em conta cada transtorno específico. É grande o número de transtornos cujas freqüências são muito diferentes num e noutro sexo.

Levando-se em conta todos os diagnósticos, verifica-se que a prevalência foi significativamente maior $\left(X_{c}^{2}=15,04\right.$; $\mathrm{p}<0,001)$ no sexo feminino, com $23,7 \%$, contra $15,6 \%$ no sexo masculino. Santana 22 , pág. 86 lembra que esse tipo de diferença tem sido relatado por muitos autores. Entretanto, outros acham que tal diferença decorra de artefato de pesquisa. Por exemplo, o que se manifesta na mulher como neurose ficaria encoberto no homem sob a fachada de consumo de álcool e outras drogas e comportamentos "desviantes". Naturalmente que a mencionada diferença depende, sobretudo, dos critérios de diagnóstico e da exaustividade na detecção dos casos na amostra. Por essas razões, alguns estudos até encon tram maior prevalência no sexo masculino. Este é o caso do mais completo estudo que se conhece sobre prevalência de transtomos mentais, o de Hagnell ${ }^{12}$, como se observa na tabela 2 .

Em síntese, esse autor continuou o estudo de Essen-Moller e col., citados por Hagnell ${ }^{12}$, realizado na população de duas vilas do sul da Suécia. Hagnell acompanhou rigorosamente essa população ao longo de dez anos (1947 a 1957). 
Cada pessoa foi psiquiatricamente examinada nos dois períodos, e os registros hospitalares, de consultórios e de informantes-chave foram todos consultados; as pessoas que emigravam das vilas ou para elas entravam foram todas acompanhadas. O seguimento da população foi de tal forma que era praticamente impossível um caso de transtomo mental não vir a ser conhecido pelo autor.

\section{TABELA 2}

Taxas de prevaléncia de transtornos mentais em populaçäo de 2.970 pessoas com dez ou mais anos de idade, examinada pessoalmente e todas acompanhadas por dez anos (1947.1957) Taxas em percentual, distribuidas por sexo e segundo as pessoas permaneceram na regiäo do estudo, ou mudaram ou en traram

\begin{tabular}{|c|c|c|c|c|c|c|c|c|c|}
\hline \multirow[b]{2}{*}{ Dragnós tico } & \multicolumn{4}{|c|}{ Masculıno $N=1526$} & \multicolumn{4}{|c|}{ Feminino $N=1444$} & \multirow[b]{2}{*}{$\begin{array}{c}\text { Total } \\
N=2970 \\
\%\end{array}$} \\
\hline & $\begin{array}{c}\text { Permaneceram } \\
N=824 \\
\%\end{array}$ & $\begin{array}{c}\text { Emugraram } \\
\mathrm{N}=360 \\
\%\end{array}$ & $\begin{array}{c}\text { Entraram } \\
\mathrm{N}=342 \\
\%\end{array}$ & $\begin{array}{c}\text { Subtotal } \\
\begin{array}{c}N=1526 \\
\%\end{array}\end{array}$ & $\begin{array}{c}\text { Permaneceram } \\
N=775 \\
\%\end{array}$ & $\begin{array}{c}\text { Emıgraram } \\
N=338 \\
\%\end{array}$ & $\begin{array}{c}\text { Entraram } \\
N=331 \\
\%\end{array}$ & $\begin{array}{c}\text { Subtotal } \\
\begin{array}{c}N=1444 \\
\%\end{array}\end{array}$ & \\
\hline $\begin{array}{l}\text { Psiconeurose } \\
\text { (Incl. Neurose Senl) }\end{array}$ & 7,9 & 7,8 & 10,0 & 8,3 & 16,5 & 20,4 & 19,9 & 18,2 & 13,1 \\
\hline $\begin{array}{l}\text { Psicose } \\
\text { (Incl. Psicose Senil) }\end{array}$ & 1,7 & 0,3 & 1,5 & 1,3 & 2,2 & 1,8 & 2,1 & 2,1 & 1,7 \\
\hline Sindr. Cerebral Organ & 1,7 & 0,6 & 1,5 & 1,4 & 0,4 & 0.0 & 0,6 & 0,3 & 0.9 \\
\hline Alcoolsmo & 9,1 & 6,1 & 6.7 & 7,8 & 0,1 & 0,0 & 0,0 & 0,05 & 4,1 \\
\hline Personalidade Psicopat. & 3,0 & 7.5 & 10,2 & 5,7 & 1,2 & 3,3 & 3,3 & 2,2 & 4,0 \\
\hline Deficiência Mental & 0,9 & 0,6 & 2,9 & 1,3 & 1,6 & 0,0 & 1,5 & 1,2 & 1,2 \\
\hline Tentativa de Suscidıo & 0,4 & 0,3 & 1,2 & 0,6 & 0,4 & 1,2 & 0.6 & 0,6 & 0,6 \\
\hline Total & 24,7 & 23,2 & 34,0 & 26,4 & 22,4 & 26,7 & 28,0 & 24,7 & 25,6 \\
\hline
\end{tabular}

Fonte: Adaptação de tabela de Hagnell 12 , pág. 41

- Taxas calculadas a partur dos casos (numerador) obitidos pela aplicação das taxas dos que permaneceram, emigraram ou entraram, sobre os respectıvos valores absolutos.

O conceito de caso foi o da psiquiatria clássica, sendo que os diagnósticos foram "checados" pelo próprio Hagnell, a partir das consultas a psiquiatras ou a outros especialistas, internação em hospital psiquiátrico e os "suspeitos" por outras fontes informantes-chave. Claro que o exame de toda uma população em dois períodos, acrescido do exame de qualquer suspeito nesse intervalo, tem chances de incluir praticamente todos os casos. Pode-se fazer apenas uma objeção a esse famoso estudo: a filiação de Hagnell à escola clássica da psiquiatria, sem que seja utilizado algum meio de validação dos diagnósticos atribuídos por esse autor.

Os resultados constantes na tabela são da maior importância, os quais podem ser considerados padrões. As prevalências dos/sexos masculino e feminino - 26,4 e $24,7 \%$, respectivamente -, não têm diferença significativa. Mas há diferenças notórias e esperadas quanto a sexo: esmagadora predominância do alcoolismo nos homens e, em menor grau, entre estes a taxa de personalidade psicopática é também mais elevada; no sexo feminino, predominaram as taxas de neurose e de psicoses. Deve-se ressaltar, porém, que nos resultados de Hagnell a taxa de esquizofrenia foi prati- 
camente a mesma nos dois sexos; a diferença decorreu de maior freqüência de depressão psicótica senil e de outras psicoses no sexo feminino. Retirando-se estas, a taxa de psicose em ambos os sexos foi de $0,98 \%$.

Além das taxas de prevalência, esse estudo fornece várias outras taxas da maior importância, entre as quais destacamse:

a) No período de dez anos, as taxas de primeira internação psiquiátrica para os sexos masculino e feminino foram de 0,5 a $0,7 \%$, respectivamente;

b) Taxas de incidência. $\mathrm{O}$ primeiro autor a mensurar essas taxas na área psiquiátrica foi Hagnell. Não as apresentamos porque elas exigem uma longa argumentação, além dos objetivos do presente trabalho; $\mathrm{e}$

c) por fim, Hagnell enfatiza que a maioria dos transtornos por ele diagnosticados foram de comprometimento leve e de curta duração (menos de quatro meses), e de natureza não-psicótica, os quais não chegaram a consultar um psiquiatra. Nesse contexto, importa destacar que, nos resultados da tabela, mais da metade da prevalência total foi devida a neuroses.

Voltando aos estudos brasileiros que estimaram prevalência de transtornos mentais, além do já comentado trabalho de Santana ${ }^{22}$, ainda há quatro estudos. Coutinho ${ }^{9}$ também estudou um bairro de Salvador, encontrando a elevada prevalência total de $49,3 \%$; porém, quase $95 \%$ dessa prevalência correram por conta de neuroses e alcoolismo, pois cada um desses transtornos apresentou uma taxa de $22,6 \%$. Como esse estudo foi realizado em população marginal, as elevadas taxas encontradas pelo autor são perfeitamente esperadas, pois nessas regiões acumulam-se particularmente os alcoolistas e neuróticos graves.

O estudo de Moura Fé e col. ${ }^{18}$ consistiu de um amplo inquérito epidemiológico em amostra representativa da população de Messejana - vizinha cidade de Fortaleza, Ceará. Seus resultados mais valiosos dizem respeito às atitudes da população em relação ao "doente mental". Mensuraram essas atitudes e a freqüência do que aquela população chama de "louco, alienado, sem juízo" etc..., encontrando uma prevalência total de $4,0 \%$. É altamente provável que tal prevalência seja absolutamente verdadeira; acima, comentamos o famoso estudo de Hagnell, onde a taxa de transtornos graves nos sexos masculino e feminino foi de $3,0 \%$ e $4,0 \%$, respectivamente.

O trabalho de Caetano ${ }^{6}$ é voltado para a epidemiologia da assistência psiquiátrica do Brasil como um todo, de 1960 a 1974. Utilizou dados da DINSAM, coletados anualmente 
mediante envio de questionário a todos os hospitais psiquiátricos do país, obtendo-se uma resposta de $80,0 \%$. Entre os resultados desse trabalho, destacam-se as taxas de primeira internação por idade, sexo e diagnóstico psiquiátrico, ao longo de 14 anos. A taxa total de primeira internação, para um e outro sexo, no mencionado período, foi excessivamente elevada entre 1966-1972, particularmente entre os homens de raça branca, entre 30 e 49 anos de idade. Entre os homens, a partir de 1966, as taxas foram sistematicamente acima de $1 \%$. habitantes e, entre as mulheres, acima de $0,8 \%$. Entre os homens, de longe, as taxas mais elevadas foram de esquizofrenia, alcoolismo e farmaco-dependência; entre as mulheres, esquizofrenia, neuroses e transtornos da personalidade.

Naturalmente que uma judiciosa interpretação dos dados de Caetano ainda é um "assunto pendente". Se o grande aumento de primeiras internações entre 1966-1972 não decorreu de uma melhora do sistema de registros, isto é, se o aumento foi de fato real, aguarda-se um esclarecimento de sua estrutura geradora. Caso isso não seja feito, os referidos dados simplesmente serão relegados à memória psiquiátrica nacional.

Tal como o anterior, o estudo de Morgado ${ }^{17}$ fornece prevalência seletiva. Basicamente, o estudo de Morgado consistiu de entrevista da mãe e/ou do pai de 150 pacientes consumidores de drogas.

Entre outros dados, nessa entrevista com os genitores dos pacientes, procurou-se conhecer a morbidade psiquiátrica em cada família. Os resultados para as 150 famílias encontram-se na tabela 3, excluindo-se delas os 150 casos-índice; se estes fossem incluídos, é óbvio que seria uma morbidade acrescentada, devido à prévia escolha daqueles 150 pacientes.

Nesse estudo, convencionou-se chamar diagnóstico provável, pois o transtorno foi diagnosticado com base nas informações da entrevista da familia e na entrevista do casoindice (o consumidor de drogas). Embora essa dupla fonte desse informações suficientes para formular esse ou aquele diagnóstico, o autor entendeu que não se podia efetuar um diagnóstico psiquiátrico sem examinar diretamente cada familiar com diagnóstico provável.

Ao entrevistar-se cada uma das 150 familias, várias vezes a entrevista foi casualmente conduzida sob forma direta, pois o próprio informante era um diagnóstico provável em geral a mãe com neurose, ou o pai com transtorno associado ao álcool. Claro, portanto, que ocorreram dois vieses no estudo: em muitos pais e mães, o diagnóstico foi direto (entrevista com o próprio) e indireto - entrevista com o cônjuge não-caso e também a entrevista individual com o filho consumidor de drogas; o segundo viés foi o fato de a 
entrevista com a familia ter sido com o pai e/ou mãe - o que pode ter levado a diagnosticar mais transtornos neles do que em seus filhos. À primeira vista, isto seria responsável pela baixa prevalência nos irmãos $(16,2 \%)$ dos 150 pacientes, relativamente às prevalências em suas mães $(24,2 \%)$ e seus pais (46,6\%). Todavia, já está bem assentado que a prevalência de transtomos mentais vai se acumulando com a idade.

\section{TABELA 3}

Morbidade psiquiatrica entre os familiares dos $150^{*}$ pacientes consumidores de drogas. Distribuiçẫo segundo o diagnóstico provável (pelos critérios da pesquisa), relativo aos pais elou irmãos dos pacientes

\begin{tabular}{|c|c|c|c|c|c|c|c|c|}
\hline \multirow[t]{3}{*}{$\begin{array}{l}\text { Diagnóstico } \\
\text { Provável }\end{array}$} & \multicolumn{2}{|c|}{$\begin{array}{l}\text { Nos Pais } \\
N=146^{* *}\end{array}$} & \multicolumn{2}{|c|}{$\begin{array}{l}\text { Nas Mães } \\
N=149^{* *}\end{array}$} & \multicolumn{2}{|c|}{$\begin{array}{l}\text { Nos Irmãos } \\
N=501\end{array}$} & \multicolumn{2}{|c|}{$\begin{array}{c}\text { Todos os } \\
\text { Familiares } \\
\mathrm{N}=796\end{array}$} \\
\hline & \multicolumn{2}{|c|}{ COM T. M. } & \multicolumn{2}{|c|}{ COM T. M. } & \multicolumn{2}{|c|}{ COM T. M. } & \multicolumn{2}{|c|}{ COM T. M. } \\
\hline & No & $\%$ & No & $\%$ & No & $\%$ & № & $\%$ \\
\hline Transtor. Assoc. ao & & & & & & & & \\
\hline $\begin{array}{l}\text { Älcool } \\
\text { Depend. de Outras }\end{array}$ & 50 & 34,2 & 4 & 2,7 & 13 & 2,6 & 67 & 8,4 \\
\hline Drogas & 1 & 0,7 & 1 & 0,7 & 40 & 8,0 & 42 & 5,3 \\
\hline Psicoses & 7 & 4,8 & 10 & 6,7 & 8 & 1,6 & 25 & 3,1 \\
\hline Neuroses & 6 & 4,1 & 18 & 12,1 & 8 & 1,6 & 32 & 4,0 \\
\hline Epilepsia & - & - & 1 & 0,7 & 5 & 1,0 & 6 & 0,8 \\
\hline $\begin{array}{l}\text { Transt. Anti-Social } \\
\text { da Personalidade }\end{array}$ & 1 & 0,7 & 1 & 0,7 & 5 & 1,0 & 7 & 0,9 \\
\hline Outros Diagnósticos & 3 & 2,0 & 1 & 0,7 & 2 & 0,4 & 6 & 0,8 \\
\hline TOTAL & 68 & 46,6 & 36 & 24,2 & 81 & 16,2 & 185 & 23,2 \\
\hline
\end{tabular}

* Não incluídos nos cálculos.

* Quatro pais e um mãe desconhecidos, - não incluídos nos cálculos.

Entre os dados da tabela 3, destaca-se que, da elevada prevalência de $46,6 \%$ nos pais, $73,4 \%(34,2$ em 46,6$)$ foi devida a transtornos associados ao álcool. Um subconjunto dessa prevalência talvez seja condicionado: esse estudo de familias - exatamente pela forma de seleção - condiciona maior freqüência dos transtornos associados a álcool e drogas. Exatamente por isso a taxa de dependência de drogas entre os irmãos dos 150 pacientes foi de $9,0 \%$ - e nesta cifra não foram incluídos o chamado consumo recreacional nem o esporádico. A taxa de psicoses entre os pais e as mães também está um pouco elevada - relativamente às taxas encontradas em estudos populacionais. Naturalmente que os resultados da tabela 3 não podem ser aferidos segundo as 
taxas esperadas a partir de estudos realizados na população geral.

Nos estudos de famílias, como o do presente caso, as taxas de transtomos específicos são condicionadas pelos casos-índice. Por exemplo, no clássico estudo de familias de alcoolistas, realizado por Rimmer e Chambers ${ }^{21}$, as taxas mais elevadas foram de transtomos afetivos e de alcoolismo; $\mathrm{e}$ isso foi real, porque todas as pessoas foram psiquiatricamente examinadas.

Além do estudo de Morgado ${ }^{18}$, há um outro trabalho brasileiro voltado para o estudo da família do paciente psiquiátrico. Trata-se do estudo realizado por Medeiros e col. ${ }^{16}$, comparando-se a morbidade pregressa nas famílias de 52 pacientes internados em um hospital psiquiátrico, com a morbidade nas familias de 52 pacientes não psiquiátricos, internados em enfermarias de Clínica Médica e de Clínica Cirúrgica.

Em $46(88,4 \%)$ famílias dos pacientes psiquiátricos e em $31(59,6 \%)$ familias do outro grupo, havia antecedentes de doença psiquiátrica. É difícil comentar esses resultados.

Ainda há um recente estudo brasileiro, realizado por Almeida Filho e col. ${ }^{1}$, que também fornece dados de prevalência. Trata-se de um inquérito epidemiológico em amostra de $10 \%$ da população de um bairro de Salvador. Os resultados ainda são preliminares, oriundos de método indireto, tendo-se como unidade a família; os dados foram coletados mediante entrevista de um de seus membros adultos. Entrevistaram-se 418 famílias, somando-se um total de 1.067 pessoas.

As taxas fornecidas são globais e dependem do ponto de corte na escala de presença das manifestações. Em três diferentes pontos de corte, aquelas taxas foram de 41,$2 ; 33,8$ e $24,2 \%$, das quais os subconjuntos de 11,$9 ; 9,8$ e $7,0 \%$ foram devidos a transtornos incapacitantes, enquanto os valores complementares foram de transtornos não incapacitantes. $\mathrm{O}$ que mais se destaca nesse estudo são as taxas de transtornos incapacitantes, que ficaram ao redor de $10 \%$. Levandose em conta que a amostra foi extraída da população acima de 14 anos de idade, a elevada dimensão dessas taxas é muito preocupante. No já comentado estudo de Hagnell ${ }^{12}$, as taxas de comprometimento grave no sexo masculino e feminino foram de 3 e $4 \%$, respectivamente. Naturalmente que nessa questão é provável que o conceito de incapacitação tenha interferido nos resultados. Por outro lado, sempre surge a polemica da oportunidade ocupacional para a população. Em regiões com restritas oportunidades, acaba ocorrendo uma supervalorização de certos transtornos, cujos portadores obviamente ficam desempregados por serem "incapazes". Em situações onde houvesse escassez de mão- 
de-obra, os "incapazes" seriam aproveitados. Contudo, no estudo de Moura Fé e col. ${ }^{18}$, a taxa do que a população chama de "doente mental" foi de $4 \%$. A questão do conceito de incapacitação cristaliza-se nessa taxa de $4 \%$. Em que medida a população não identifica um adicional de transtornos por serem menos óbvios?

No já comentado estudo de Almeida Filho e col. ${ }^{1}$, sugere-se que o resultado que mais se aproxima do real seja o de prevalência global de $24,2 \%$ e taxa de incapacitados por transtornos mentais de 7,8\%. Ao comparar-se esta cifra com os $4 \%$ de Moura Fé e col. ${ }^{18}$, verifica-se que uma é praticamente o dobro da outra. Não obstante isso, é muito provável que as taxas de incapacitação por doença mental nas duas populações sejam similares. Se isso é verdade, a diferença nas taxas dos dois estudos - com amostras representativas - decorreu de questões conceituais.

\section{MORBIDADE INTERNADA PROPORCIONAL POR DIAGNÓSTICO}

Esse importante indicador da epidemiologia da assistência psiquiátrica está bem dimensionado e permite acompanhar a evolução da demanda internada nos hospitais psiquiátricos do Brasil. Os estudos disponíveis que fornecem diagnósticos de grande número de pacientes na primeira internação psiquiátrica foram aqui aproveitados.

$\mathrm{Na}$ tabela 4, encontra-se parte dos dados do estudo de Araújo $^{2}$ referentes a todo o Brasil. Ao que nos consta, esse foi o primeiro trabalho de grande porte, com numerosos dados hospitalares e ambulatoriais a nível nacional.

Em primeiro lugar, observa-se que um pouco mais de $3 / 5$ do total das primeiras internações foram de pacientes do sexo masculino $(61,65 \%)$. No total por diagnóstico destaca-se que, na década de cinqüenta, o Brasil ainda tinha perto de $6 \%$ de psicoses sifilíticas e perto de $2 \%$ de psicoses por outras infecções; mais à frente, veremos que nas décadas subseqüentes essas psicoses foram rareando.

É interessante que, na distribuição dos diagnósticos por sexo, há resultados que estão de acordo com a literatura, mas, alguns dão margem a dúvidas. A grande predominância das psicoses sifilíticas e exotóxicas (quase todas por alcoolismo) no sexo masculino e das psicoses endotóxicas no sexo feminino (pelo componente associado ao parto e ao aborto séptico) é perfeitamente esperada. Mas é difícil explicar a grande diferença em psicoses por lesões cerebrais, oligofrenias e epilepsias, pois são transtornos que não têm diferenças substantivas quanto ao sexo. Por outro lado, esperar-se-ia encontrar maiores proporções dos diagnósticos de PMD e de neuroses no sexo feminino. 
Os dados constantes na tabela 5 são mais instrutivos que os da 4 , pois eles evidenciam os diagnósticos em dois sentidos: histórico-evolutivo e diferenças no âmbito institucional. As duas primeiras colunas dizem respeito aos dados consolidados de primeiras internações de praticamente todo o Brasil; a terceira coluna refere-se às primeiras internações de um grande hospital público do Estado de São Paulo, o de Juqueri; os dados da quarta, quinta, sexta e sétima colunas são de dois hospitais particulares conveniados da cidade de São Paulo: Instituto Achê e Sanatório Bela Vista.

\section{TABELA 4}

Pacientes de primeira internação psiquiátrica em hospitais públicos e particulares do Brasil, no ano de 1952, distribuição por sexo e segundo o diagnóstico psiquiátrico.

\begin{tabular}{|c|c|c|c|c|c|c|}
\hline \multirow[b]{2}{*}{ Diagnóstico } & \multicolumn{2}{|c|}{ Masculino } & \multicolumn{2}{|c|}{ Feminino } & \multicolumn{2}{|c|}{ Total } \\
\hline & Nọ & $\%$ & Nọ & $\%$ & No & $\%$ \\
\hline Psicoses por infecc. infestac. & 261 & 47,54 & 288 & 52,46 & 549 & 1,91 \\
\hline Psicoses Sifilíticas & 1293 & 80,86 & 306 & 19,14 & 1599 & 5,57 \\
\hline Psicoses Exotóxicas & 2809 & 89,01 & 347 & 10,99 & 3156 & 10,99 \\
\hline Psicoses Endotóxicas & 385 & 37,82 & 633 & 62,18 & 1018 & 3,54 \\
\hline Psicoses por Lesões Cerebrais & 953 & 75,69 & 306 & 24,31 & 1259 & 4,38 \\
\hline Oligófrenias & 1410 & 62,58 & 843 & 37,42 & 22,53 & 7,84 \\
\hline Epilepsias & 1444 & 65,91 & 747 & 34,09 & 2191 & 7,63 \\
\hline Esquizofrenias & 4546 & 57,01 & 3428 & 42,99 & 7974 & 27,77 \\
\hline P. M. D. & 1459 & 48,73 & 1535 & 51,27 & 2994 & 10,42 \\
\hline Psicopatias mistas e assoc. & 198 & 62,66 & 118 & 37,34 & 316 & 1,10 \\
\hline Psicoses Psicogênicas & 349 & 48,95 & 364 & 51,05 & 713 & 2,48 \\
\hline Neuroses & 722 & 46,94 & 816 & 53,06 & 1538 & 5,36 \\
\hline Person. Psicopata & 515 & 60,73 & 333 & 39,27 & 848 & 2,95 \\
\hline Transtornos não classificados & 1430 & 61,77 & 885 & 38,23 & 23,15 & 8,06 \\
\hline Sem Perturbaçāo Mental & 428 & 56,24 & 333 & 43,76 & $761^{*}$ & - \\
\hline Total & 18.202 & 61,74 & 11,615 & 38,26 & 29,484 & 100,0 \\
\hline
\end{tabular}

Fonte: Reanálise de parte dos dados de Araújo ${ }^{2}$.

* Não incluído no cálculo das percentagens do to tal.

A tabela 5 resume os resultados totais dos trabalhos de Araújo $^{2}$, Botelho ${ }^{5}$, Pinto Cesar ${ }^{20}$ e Cardoso e col. ${ }^{7}$. Embora em seus originais se detectem algumas diferenças na terminologia dos transtomos psiquiátricos, os resultados de todos podem ser expressos sob as categorias da Classificação Brasileira de Doenças Mentais. Desses quatro trabalhos, apenas o de Pinto Cesar ${ }^{20}$ contém diferenças importantes quanto ao diagnóstico. Para aproveitar seu precioso trabalho, decidiu-se que as frações correspondentes à parafrenia e à paranóia fossem incluídas no grupo das esquizofrenias, que psicoses autotóxicas correspondessem a psicoses endotóxicas, e paralisia geral a psicoses sifilíticas. Em termos metodológicos, essas aproximações são válidas, pois se não há 
absoluta equivalência (superposição) entre as duas denominações, a área de intercessão entre elas é grande. Mesmo que não haja superposição entre duas categorias aparentemente diferentes, se há intercessão entre elas, e se o seu montante em relação ao conjunto total não é grande, a aproximação não encerra erro importante. No original de Pinto Cesar, as proporções de parafrenia, paranóia e paralisia geral são sempre inferiores a $5 \%$. Por outro lado, o conhecimento dá respaldo, em termos da natureza do transtorno, para as respectivas inclusões nas categorias da Classificação Brasileira de Doenças Mentais, - empregada nos três outros estudos citados acima. Essas considerações visam a legitimar o aproveitamento dos dados de 1940 de Pinto Cesar ${ }^{20}$, porque é o primeiro trabalho nacional com grande número de primeiras internações psiquiátricas. Além disso, desses quatro trabalhos, é o que dá a maior coerência intema aos dados. Legitimado tal aproveitamento, os dados da tabela 5 e 6 dão respaldo empírico para a interpretação da morbidade proporcional internada por quase quatro décadas.

Naturalmente que nesse tipo de análise de dados sempre há questões incontornáveis. Na tabela 5 , observa-se que psicoses mistas e associadas e psicoses psicogênicas não foram contempladas nos dois estudos de São Paulo, e o inverso no diagnóstico de psicose de involução. Isso é uma prova irrefutável de que a Classificação Brasileira de Doenças Mentais de fato não era aceita na íntegra. É curioso que Cardoso e col. ${ }^{7}$, que fornecem valiosos dados ao longo de quatro qüinqüênios, explicitem a adoção da mencionada classificação. Não fôra Pinto Cesar ${ }^{20}$ também empregar o diagnóstico de psicose de involução, - mas encontrar apenas $3 \%$ em um hospital público - seria praticamente impossível aproveitar o conjunto dos dados de Cardoso e col. ${ }^{7}$. Isto porque estes autores dão altas proporções no grupo de psicoses de involução: se não tivesse um parâmetro como o de Pinto Cesar, essas altas proporções necessariamente condicionariam apreciável redução nos outros grupos de diagnóstico. Claro que a grande diferença desses dois trabalhos quanto ao diagnóstico de psicose de involução é devida ao contexto institucional: os dados de um são oriundos de um hospital público, onde os pacientes carentes e mais graves são internados, enquanto os dados do outro são oriundos de dois hospitais particulares conveniados, com importante conjunto dos leitos destinados à psiquiatria geriátrica e a "repouso".

$\mathrm{O}$ referido contexto institucional também explica algumas diferenças em certos grupos de diagnósticos comuns aos quatro estudos da tabela 5 . Os grupos de psicoses exotóxicas (a maioria por alcoolismo) e de neuroses são sistematicamente altos nas 4ạ, 5ạ, 6ạ e 7ạ colunas, ao passo que, nestas, as proporções dos grupos de psicoses sifilíticas, 
psicoses endotóxicas, oligofrenias e esquizofrenias são sistematicamente bem mais baixas que as respectivas proporções nas três primeiras colunas. Claro que os últimos quatro grupos de transtornos psiquiátricos exigem maiores cuidados de abordagem e têm pequena chance de alta a curto prazo; é precisamente por isso que eles acabam se concentrando nos serviços assistenciais que originam os dados das três primeiras colunas da tabela 5 . Essas diferenças são mais nítidas entre os dois estudos de São Paulo, isto é, entre a 3ạ e as $4 a ̣, 5 a ̣, 6 a$ e $7 a ̣$ colunas. As proporções das últimas quatro colunas não diferem tanto daquelas das duas primeiras, porque os dados do Brasil incluem um subconjunto de dados oriundos de hospitais particulares conveniados que, embora pequeno, reduz um pouco as diferenças.

$\mathrm{O}$ indicador de morbidade proporcional, restrito às primeiras internações, mostra que esquizof renia e alcoolismo são o carro-chefe dos hospitais psiquiátricos do país. Entretanto, destaca-se que, na rede particular-conveniada, como indicam as colunas 4, 5, 6 e 7 da tabela 5, as internações por neurose têm alcançado níveis excessivos, ultrapassando $20 \%$ do total de pacientes. Naturalmente que, entre outras coisas, isso decorre da não-disponibilidade de uma boa rede de atendimento ao nível de ambulatório.

\section{TABELA 5}

Pacientes de primeira internação psiquiátrica no Brasil como um todo, e em dois hospitais de São Paulo, distribuidos segundo a proporção de cada diagnóstico (em relação ao total de primeiras internaçōes)

\begin{tabular}{|c|c|c|c|c|c|c|c|}
\hline $\begin{array}{r}\text { Tamanho, Local e } \\
\text { Data }\end{array}$ & $\begin{array}{c}N=28660 \\
\text { Brasil } \\
1952 \text { (a) }\end{array}$ & $\begin{array}{c}\mathrm{N}=34252 \\
\text { Brasil } \\
1953(\mathrm{~b})\end{array}$ & $\begin{array}{l}N=5258 \\
\text { São Paulo } \\
1940(\mathrm{c})\end{array}$ & $\begin{array}{c}N=2127 \\
\text { São Paulo } \\
1946-50 \\
\text { (d) } \\
\%\end{array}$ & $\begin{array}{c}\mathrm{N}=2657 \\
\text { São Paulo } \\
1951-55 \\
\text { (d) } \\
\%\end{array}$ & $\begin{array}{c}\mathrm{N}=2726 \\
\text { São Paulo } \\
1956-60 \\
\text { (d) } \\
\%\end{array}$ & $\begin{array}{c}N=3236 \\
\text { São Paulo } \\
1961-65 \\
\text { (d) } \\
\%\end{array}$ \\
\hline Psicoses por infec. infest. & 1,91 & 1,80 & 0,70 & 0,90 & 0,80 & 0,62 & 0,43 \\
\hline Psicoses Sifilíticas & 5,57 & 5,40 & 3,69 & 0,33 & 0,26 & 0,36 & 1,40 \\
\hline Psicoses Exotóxicas & 10,99 & 10,70 & 5,50 & 19,32 & 19,24 & 19,62 & 19,10 \\
\hline Psicoses Endotóxıcas & 3,54 & 3,40 & 6,85 & 5,55 & 2,30 & 0,81 & 0,24 \\
\hline Psicoses por Lesões Cerebrals & 4,38 & 5,50 & 6,73 & 11,05 & 9,25 & 9,31 & 9,64 \\
\hline Oligof renıas & 7,84 & 7,50 & 13,96 & 0,70 & 0,26 & 0,20 & 0,06 \\
\hline Epilepsias & 7,63 & 7,50 & 10,12 & 3,20 & 10,61 & 10,52 & 8,40 \\
\hline Esquizof renias & 27,77 & 27,30 & 37,87 & 12,56 & 9,73 & 8,71 & 5,85 \\
\hline P. M. D. & 10,42 & 10,20 & 6,69 & 3,10 & 3,42 & 3,10 & 2,71 \\
\hline Psicoses Mistas e Assoc. & 1,10 & 1,00 & - & - & - & - & - \\
\hline Psicoses Psicogênicas & 2,48 & 2,40 & - & - & - & - & - \\
\hline Neuroses & 5,36 & 5,50 & 1,05 & 16,17 & 17,72 & 22,67 & 26,11 \\
\hline Personalidade Psicopat. & 2,95 & 2,90 & 3,84 & 10,06 & 7,80 & 6,45 & 3,99 \\
\hline Psıcose de Involução & - & - & 3,00 & 17,06 & 18,51 & 17,64 & 22,07 \\
\hline Não Classificados & 8,06 & 7,9 & - & - & - & - & - \\
\hline Total & 100,00 & 100,00 & 100,00 & 100,00 & 100,00 & 100,00 & 100,00 \\
\hline
\end{tabular}

Fonte: a) Araújo ; b) Botelho ; c) Pinto Cesar ${ }^{20}$ e d) Cardoso e col. ${ }^{7}$. 
Os dados da tabela 6 poderiam ser apresentados sob a forma de total na tabela 5, mas, por enquanto, isso só é possível para esquizofrenia, alcoolismo e farmaco-dependência - equivalendo à psicoses exotóxicas -, e deficiência mental ou oligofrenia. Tal aproximação dessas duas últimas tabelas exigiria o remanejamento de todos os grupos divergentes para a categoria de "outros diagnósticos", o que significa perder preciosas informações de vários grupos específicos. Poder-se-ia afirmar, por exemplo, que os valores para psicoses afetivas na tabela 6 estão de acordo com o esperado para a PMD e que, portanto, a equivalência é legítima; isso é verdade, mas seria meio precipitado equivalê-las sem verificar quais foram os dados brutos que originaram o grupo de psicoses afetivas da tabela 6. E esse tipo de dado não está facilmente disponível.

Ao se compararem os grupos de diagnósticos de esquizofrenia, alcoolismo mais farmaco-dependência e deficiência mental, constantes na tabela 6 , com as respectivas categorias das duas primeiras colunas da tabela 5, observam-se mudanças importantes. Nas duas décadas (1952 a 1974), houve apreciável redução de deficiência mental ou oligofrenia e grande aumento de alcoolismo mais farmaco-dependência ou psicoses exotóxicas; as proporções de esquizofrenia permaneceram estacionárias. Contudo, no global, houve grande aumento da demanda, porque os valores de $\mathrm{N}$ aumentaram muito de 1952 a 1974.

Os dados da tabela 6 mostraram conhecidas diferenças por sexo: predomínio de alcoolismo e farmaco-dependência no sexo masculino e o oposto nos grupos de psicoses afetivas e de neuroses e transtomos da personalidade. Nessa tabela, introduzimos os dados totais de Madalena ${ }^{14}$, referentes às primeiras internações nos anos de 1976/77 em um hospital psiquiátrico da cidade do Rio de Janeiro. Em seus dados, destaca-se a elevada proporção de diagnóstico de esquizofrenia.

Com os dados das tabelas 5 e 6 , observa-se que as primeiras internações nos hospitais psiquiátricos do Brasil vêm aumentando. Em 1952, 1953, 1960, 1970 e 1974, os totais de primeiras internações foram de $28.660 ; 34.252 ; 46.735$; 138.786 e 108.975 , respectivamente. Há um significativo excesso em 1970, o que fortemente sugere problemas na coleta dos dados.

Em relação aos valores absolutos de primeiras internações das tabelas 4, 5 e 6 (valor $\mathrm{N}$ ), há evidências de que não se referem a todos os hospitais psiquiátricos, públicos e particulares, do Brasil. Em seu trabalho, Caetano ${ }^{6}$ esclarece que, à exceção do ano de 1974 , mais de $80 \%$ dos hospitais psiquiátricos do país remetem para a DINSAM o questionário preenchido com os dados brutos. As informações das 
últimas três tabelas, com dados para todo o país, são, pois, de suma importância.

\section{TABELA 6}

Primeiras internações em hospitais psiquiátricos do Brasil, em quatro periodos distintos, distribuidos por grupo de diagnóstico e por sexo.



Fonte: "Dados da DINSAM, extraídos de Caetano ${ }^{6} ;{ }^{* *}$ dados extraídos de Madalena ${ }^{14}$. ${ }^{* * *}$ Masc. $=24,6 \% ;$ Fem. $=2,5 \%$.

Além dos estudos acima, dos quais apresentamos parte de seus resultados, há outros estudos nacionais que fornecem o indicador de morbidade proporcional por diagnóstico. De Pernambuco há importantes dados nos trabalhos de Pernambucano e Di Lasco ${ }^{19}$, de Lima Neto $^{13}$ e num recente de Assunção Filho e col. ${ }^{3}$. Do Rio de Janeiro há o estudo de Madalena ${ }^{14}$, onde, além do referido indicador, os dados estão distribuídos por outras variáveis de grande interesse, tais como ocupação, situação previdenciária, idade e sexo, etc ... Com pacientes atendidos em ambulatórios, há o trabalho de Frota Pinto ${ }^{11}$ e o de Valente ${ }^{24}$. Este último faz também um bom inventário das condições gerais de vida dos pacientes estudados. Seus dados, juntamente com os de Frota Pinto ${ }^{11}$, inauguram outra linha de investigação em nosso meio - a da epidemiologia da assistência psiquiátrica a nivel ambulatorial. Neste sentido, é importante resgatar os dados de Araújo $^{2}$, referentes à rede ambulatorial do antigo Serviço Nacional de Doenças Mentais.

\section{COMENTÁRIO FINAL}

É inegável que os dados mais relevantes do presente trabalho são os relativos às primeiras intemações em hospitais 
psiquiátricos do Brasil. Seus valores aumentaram progressiva e constantemente no período estudado. Todavia, ainda não é possível saber se tal aumento é real, uma vez que a população do país também tem crescido constantemente. De qualquer forma, mesmo que os dois referidos aumentos sejam paralelos, isso não significa que a estrutura assistencial continue inalterada. Nas grandes cidades, crescem a densidade populacional, o desemprego, pobreza, tensão e violência que, entre outras aflições, geram um aumento das internações em hospitais psiquiátricos. Em tais condições, não há como evitar que um crescente subconjunto populacional passe algum tempo no hospital psiquiátrico.

$\mathrm{O}$ atendimento à demanda psiquiátrica, entretanto, está muito longe de escolha por parte da clientela: a demanda é dirigida. Os dados da tabela 5 mostram em números aquilo que empiricamente já se sabia: os pacientes mais graves, com pior prognóstico e que conseqüentemente demandam internações muito longas, se concentram em hospitais públicos, enquanto aqueles com intemação mais curta, em geral vão para os hospitais particulares conveniados. De certa forma, não poderia ser diferente, já que os pacientes graves, de longos períodos de internação, são pacientes mais caros ao hospital. Ao priorizarem a intemação dos pacientes menos graves, os hospitais particulares conveniados buscam a obtenção de taxas de lucro mais elevadas, cabendo aos hospitais públicos o ônus das internações prolongadas.

As colunas 4, 5, 6 e 7 da tabela 5 dizem respeito à demanda particular-conveniada; é da maior importância que em duas décadas as internações por neuroses tenham sido tão elevadas. Certamente que, em tais condições, é um sonho pensar em ambulatório para tratar os pacientes graves. Ora, se se recorre à internação para o tratamento de neuróticos, é um irrealismo falar em ambulatório de saúde mental. Isso está coerente com o trabalho de Frota Pinto ${ }^{11}$, onde se verificou que aproximadamente $70 \%$ de 25.000 pacientes atendidos em ambulatọ́rio "não são portadores de verdadeiras doenças mentais e sim de socioses". Há, portanto, o risco de um subnivelamento de todo o conjunto assistencial: o ambulatório atende a problema social, o hospital particular-conveniado interna pacientes que seriam do ambulatório e o hospital público é transformado em custódia.

Como se sabe, a maior parte dos pacientes graves tem ficado sob tutela direta do Estado, desde a época do magnânimo Dom Pedro II. Com a criação da Previdência Social, ocorreu a primeira modificação, onde os pacientes segurados passaram a poder ser internados na rede conveniada. Essa previdência construiu hospitais até para especialidades 
sofisticadas, mas para sua grande demanda psiquiátrica limitou-se a implantar uma precária rede de triagem e encaminhamento de seus pacientes para a rede conveniada; de fato, ela não patrocinou a criação de nenhum serviço que zele e cuide do segurado psiquicamente enfermo.

A partir de novembro de 1983, ocorreu outra modificação: os pacientes da Previdência Social passaram a ser atendidos ou internados naqueles hospitais públicos do antigo Serviço Nacional de Doenças Mentais. Naturalmente que tal repasse de encargos é cheio de conseqüências, as quais estão longe de um razoável encaminhamento. Não se sabe, por exemplo, nem mesmo a estatística referente à demanda de pacientes segurados. Só há um trabalho, o de Cerqueira e col. ${ }^{8}$, que fornece dados para discutir algumas consequências.

Há problemas reais que culminam em dificuldades imensas: a efetiva organização e atualização dos serviços e hospitais; a formação de recursos humanos e sua incorporação aos serviços e hospitais; a estrutura de cargos e salários - o corpo técnico desses serviços e hospitais públicós ganha muito menos do que os profissionais da Previdência Social. Caso não se encaminhe bem a solução dessas dificuldades, o paciente psiquicamente enfermo não será bem assistido. Os dados disponíveis, como os constantes nos trabalhos de Araújo $^{2}$ e Botelho ${ }^{5}$, demonstram que, na década de cinqüenta, havia uma bem coordenada estrutura nacional para cuidar daqueles pacientes.

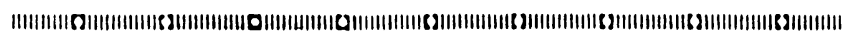

The authors present in this paper three types of data on mental disorders: prevalence rates in representative samples, prevalence rates (primary data) in a family study and the indicators of diagnostics' proportion among the patients admitted to Brazilian psychiatric hospitals.

Concerning to one survey carried out over representative samples, the overall prevalence rate was $20 \%$, of which, $3.0 \%, 14.6 \%, 0.7 \%, 1.1 \%$, and $0.5 \%$ were due respectively to alcoholism, neurosis, psychosis, mental deficiency, and organic brain syndrome. The sex distribution has shown an overt but expected excess of alcoholism among the males, and neurosis among the females.

In one study related to the original family (parents and siblings) of 150 heavy drug abusers, the overall prevalence rate of mental disorders among 796 relatives of these 150 drug users was $23.2 \%$, of which, $8.4 \%, 5.3 \%, 3.1 \%, 4.0 \%$, $0.8 \%, 0.9 \%$, and $0.8 \%$ were respectively due to alcohol related problems, drug addiction, psychosis, neurosis, epilepsy, antisocial personality and other diagnosis. The 
alcohol related problems were by far the most frequent among the fathers, drug addiction among the siblings, and neurosis among the mothers of those 150 index-patients.

The characteristics of Brazil's psychiatric facilities are partially viewed by means of first admittion's indicator, at psychiatric wards. The proportion through the diagnosis is provided in a time-distribution table from 1940 to 1977. Such data shows that the first admittion rate has increased in the last four decades; the most frequent diagnosis have been schizophrenia, alcoholism and drug addiction.

However, between the diagnostic profile of public hospitals and that of private ones, there is an outstanding difference. The former with an increased amount of serious disorders, while the latter shows a great number of lighters ones. Such findings are discussed with an aim of helping Mental Health Planning.

\section{REFERÊNCIAS BIBLIOGRÁFICAS}

1. ALMEIDA FILHO, N.; SANTANA, V.S.; COUTINHO, D.M.; AGUIAR, W. \& ANTONISSEN, D. Prevalência de desordens mentais em uma área industrial da região metropolitana de Salvador: aspectos metodológicos e achados preliminares. Universitas, Salvador, 32: 59-72, 1985.

2. ARAÚJO, D. Atividades da seção de cooperação do SNDM: dados estatísticos. Arq. Serv. Nac. Doenç. Ment., 4 (4): 247-51, 1955.

3. ASSUNÇÃO FILHO, A.; AMORIM, E.M.S.; CUNHA, M.C. \& SOUGEY, E.B. Estudo nosográfico de uma população hospitalar no nordeste brasileiro. Neurobiol., 47 (4): 243-60, 1984.

4. BLUM, R.H. Case identification in psychiatric epidemiology: methods and problems. Milb. Mem. Fund. Quart., 40: 25388, 1962.

5. BOTELHO, A. Atividades do Serviço Nacional de Doenças Mentais em 1951, 1952 e 1953. Arq. Serv. Nac. D. Ment., 4 (4): 192-246, 1955.

6. CAETANO, R. Admisiones de primer ingreso a los servicios psiquiátricos en Brasil. Bol. Of. Sanit. Panam., 92 (2): 103-17, 1982.

7. CARDOSO, W.; OLIVEIRA NETO, E.M. \& SOLINGER, L.A.C. Contribuição para o estudo da incidência das doenças mentais na cidade de São Paulo. Bol. Centro Est. Franco da Rocha, 4 (3): 7-10, 1968.

8. CERQUEIRA, L. et alii. Uma avaliação do sistema AmbulatórioHospital Psiquiátrico do INPS no qüinqüênio $1971 / 75$. R. Assoc. Bras. Psiquiat., 2 (1): 19-64, 1980.

9. COUTINHO, D.M. Prevalência de doenças mentais em uma comunidade marginal: um estudo do Maciel, Salvador. Salvador, 1976. [Tese de Mestrado em Medicina Interna Faculdade de Medicina - UFBa] . 
10. DOHRENWEND, B.P. \& DOHRENWEND, B.S. The problem of validity in field studies of psychological discorders. J. $A b n$. Psychol., 70 (1):52-69, 1965.

11. FROTA PINTO, G. Prevalência dos distúrbios psiquiátricosociais no município de Fortaleza. R. Assoc. Bras. Psiquiat., 1 (1): 27-31, 1977.

12. HAGNELL, $O$. A prospective study of the incidence of mental disorders. Sweden, Svenska Bokförlaget, Norstedts, Scan. Univ. Books, 1966. 175 p.

13. LIMA NETO, L.I.A. Contribuição ao estudo da distribuição geográfica de psicopatas em Pernambuco. Neurobiol., 14 (3): 207-15, 1951.

14. MADALENA, J.C. Uma pesquisa epidemiológica na Casa de Saúde Dr. Eiras. In: MADALENA, J.C. Epidemiologia das doenças mentais. Rio de Janeiro, Ed. do Autor, p. 23-49 1980.

15. MANIS, J.G.; BRAWER, M.J.; HUNT, C.L. \& HERCHER, L.C. Estimating the prevalence of mental illness. Am. Sociol. Rev., 29: 84-9, 1964.

16. MEDEIROS, T.A.; OLIVEIRA, M.G.L. \& BARROS, J.F.S. EStudo comparativo da incidência de psicopatas em famílias de pacientes psiquiátricos e da população geral. Neurobiol., 34 (4): 187-90, 1971.

17. MORGADO, A.F. Dependência de drogas: descrição de uma pesquisa empírica e revisão de alguns aspectos relevantes para sua prevenção. São Paulo, 1983. 281 p. [Tese de Doutoramento em Medicina Preventiva - Faculdade de Medicina - USP]

18. MOURA FE, N. \& SAMPAIO, J.J.C. Pesquisa sobre epidemio. logia psiquiátrica em Messejana. Fortaleza, Secretaria de Saúde do Estado do Ceará, Imprensa Oficial, 1981. 121 p.

19. PERNAMBUCANO, U. \& DI LASCIO, A. Estudo estatístico das doenças mentais em 400 primeiros internados em casa de saúde particular. Neurobiol., 3 (4): 497-504, 1977.

20. PINTO CESAR, E. Alguns aspectos da incidência das moléstias mentais no Estado de São Paulo. Arq. Assist. Psicop. Est. S. Paulo, 8 (3/4): 299-333, 1943.

21. RIMMER, J. \& CHAMBERS, D.S. Alcoholism: methodological considerations in the study of family illness. Am. J. Ortopsychiat., 39 (5): 760-8, 1969.

22. SANTANA, V.S. Estudo epidemiológico das doenças mentais em um bairro de Salvador. Salvador, Secretaria de Saúde do Estado da Bahia, 1982. 139 p. [Série de estudos em saúde, 3] .

23. SROLE, L.; LANGNER, T.S.; MICHAEL, S.T.; OPLER, M.K. \& RENNIE, T.A. Mental health in the metropolis: the midtown study. New York, McGraw-Hill, 1962. v. 1.

24. VALENTE, M.A.P. Pesquisas sobre anormalidades psíquicas no Distrito Federal. Brasilia Med., 2 (3/4): 38-54, 1968.

25. VON ZERSSEN, D. \& WEYERER, S. Sex differences in rates of mental disorders. Int. J. Ment. Health, 11 (1/2): 9-45, 1982. 
26. WILLIANS, P.; TARNOPOLSKY, A. \& HAND, D. Case Definition and case identification in Psichiatric epidemiology Psychol. Med., 10:101-14, 1980. 